Tohoku J. Exp. Med., 2008, 214, 113-120

\title{
Effectiveness of Oseltamivir Treatment among Children with Influenza A or B Virus Infections during Four Successive Winters in Niigata City, Japan
}

\author{
Maki Sato, ${ }^{1,2}$ Reiko Saito, ${ }^{1}$ Isamu Sato, ${ }^{3}$ Naohito Tanabe, ${ }^{1}$ Yugo Shobugawa, ${ }^{1}$ \\ Asami Sasaki, ${ }^{1}$ Danjuan Li, ${ }^{1}$ Yasushi Suzuki, ${ }^{1}$ Mizuho Sato, ${ }^{1,2}$ Takatsugu Sakai, ${ }^{1}$ \\ Taeko Oguma, ${ }^{1,2}$ Hiroki Tsukada, ${ }^{2}$ Fumitake Gejyo ${ }^{2}$ and Hiroshi Suzuki ${ }^{1}$ \\ ${ }^{1}$ Department of Public Health, Niigata University Graduate School of Medical and Dental Sciences, \\ Niigata, Japan \\ ${ }^{2}$ Department of Medicine II, Niigata University Graduate School of Medical and Dental Sciences, \\ Niigata, Japan \\ ${ }^{3}$ Yoiko Pediatric Clinic, Niigata, Japan
}

Oseltamivir has been used for treatment of influenza A and B infections, but recent reports documented that it was less active against the latter. We compared the effectiveness of oseltamivir in children between laboratory confirmed influenza A and B over 4 influenza seasons from 2001 to 2005 in a pediatric clinic in Japan. Among 1,848 patients screened, 299 influenza A and 209 influenza B patients were administered oseltamivir (treated groups), and 28 influenza A and 66 influenza B patients were assigned as non-treated groups. The duration of fever, defined as period when patients had the maximum temperature higher than $37.5^{\circ} \mathrm{C}$ in three-time measurements in a day after the clinic visit, was evaluated among the four groups. In uni-variate analysis, the duration of fever was shorter for treated group than non-treated for influenza A $(1.8 \pm 0.9$ days vs $2.6 \pm 1.3$ days, $p<0.01)$, but it was not significant for influenza B $(2.4 \pm 1.3$ days vs $2.8 \pm 1.2$ days, $p=0.9)$. The fever duration was longer in treated influenza B than A patients $(p<0.01)$. Multi-variate analysis indicated younger age ( $<6$ years old) and higher body temperature at the clinic visit prolonged the duration of fever. Adjusted average duration of fever indicated that oseltamivir was effective for both types, but more effective on influenza A, and the benefit increased for younger children. Our data provide evidence that oseltamivir is beneficial for influenza infections, but the effectiveness is differed by type and age. - influenza; anti-viral drugs; oseltamivir; children; effectiveness.

Tohoku J. Exp. Med., 2008, 214 (2), 113-120.

(C) 2008 Tohoku University Medical Press

Influenza outbreaks occur annually across the world, causing excess morbidity and mortality (Simonsen et al. 2000; Nicholson et al. 2003; Centers for Disease Control and Prevention 2006). For influenza treatment, there are two types of anti-influenza drug: amantadine and neuraminidase inhibitors (oseltamivir and zanamivir) (Monto 2003; Oxford et al. 2003; Moscona 2005; Oxford 2005; Jefferson et al. 2006). Amantadine is effective for treatment of influenza

Received October 3, 2007; revision accepted for publication January 7, 2008.

Correspondence: Reiko Saito, M.D., Ph.D., Department of Public Health, Niigata University Graduate School

of Medical and Dental Sciences, 1-757, Asahimachi-dori, Chuo-Ward, Niigata City, Niigata 951-8510, Japan.

e-mail: jasmine@med.niigata-u.ac.jp 
A virus infections while neuraminidase inhibitors are for both influenza A and B (Treanor et al. 2000). Early treatment reduces the severity and duration of influenza illness and associated complications (Nicholson et al. 2000; Aoki et al. 2003; Kawai et al. 2005).

The neuraminidase inhibitors, zanamivir and oseltamivir, interfere with the release of progeny influenza viruses from infected host cells and spread to neighboring cells in the respiratory tract. Clinical efficacy of oseltamivir has been established as treatment for influenza in adults (Hayden et al. 1999; Nicholson et al. 2000; Treanor et al. 2000) and children (Whitley et al. 2001). The neuraminidase inhibitors were tested to be less active against influenza $B$ than A viruses in vitro studies (Boivin and Goyette 2002; Aoki et al. 2003; Mungall et al. 2004). Moreover, increasing evidence suggests that oseltamivir is less effective against influenza $B$ than influenza $A$ infections (Kawai et al. 2006; Sugaya et al. 2007). The present study was conducted to evaluate the efficacy of oseltamivir treatment among children with influenza $\mathrm{A}$ and $\mathrm{B}$ virus infections during four successive winters in Japan using uni-variate and multi-variate analysis adjusted for various factors that affect the course of illness.

\section{Materials ANd Methods}

\section{Study population and laboratory methods}

This study was conducted during 4 influenza seasons from November 2001 to May 2005 at a private pediatric outpatient clinic located at the city center in Niigata City, Japan with a total population of approximately 500,000. This clinic had no bed facility, and approximately 2,300 outpatients visited per month.

Influenza-like illness was defined on the basis of a sudden fever ( $\left.\geqq 37.5^{\circ} \mathrm{C}\right)$ and any acute respiratory symptoms and signs, such as, cough, rhinorrhea, sneezing, wheezing, sore throat, headache, nausea, or malaise. Nasopharyngeal swabs or aspirates were examined with rapid antigen test kits for diagnosis of influenza A or B prior to antiviral drug treatment (oseltamivir or amantadine) at the initial office visits. Influenza rapid test kits, such as QuickVue Rapid SP influ (DS Pharma Biomedical Co., Ltd., Osaka), Espline Influenza A\&B-N (Fujirebio Inc., Tokyo), and Quick S-Influ A/B "SEIKEN" (Denkaseiken Co., Ltd., Tokyo) were used to screen influenza A or B infections.

Patients were assigned to influenza treatment or non treatment groups, depending whether or not they want to receive antiviral drug medication according to the rapid test results. For patients with influenza A, the decision of whether to receive antivirals (oseltamivir or amantadine) or not was left to patients or their family. For influenza B, patients could choose either oseltamivir or no antiviral therapy. The two drugs were administered twice daily (oseltamivir, $150 \mathrm{mg}$ per day for children $\geqq 37.5 \mathrm{~kg}$ in weight; or $4 \mathrm{mg} / \mathrm{kg}$ for children with $<37.5 \mathrm{~kg}$ : amantadine, $1.5-2.5 \mathrm{mg} / \mathrm{kg}$ ). Both drugs were prescribed for 5 days. For some patients, administration of drugs was discontinued if symptoms were alleviated within 5 days. Single use of antipyretics was allowed when a child had a fever more than $38.5^{\circ} \mathrm{C}$.

Written informed consent was acquired from parents of patients to obtain clinical information and specimens for virological investigations upon enrollment to the study. Age, sex, body weight, vaccination status, use of antipyretics, type of drug, the time from the onset of fever to the administration of anti-influenza drug, body temperatures, and the results of rapid antigen test kits were recorded for all patients by the clinician at the time of report to the clinic. The parents were given a diary card to record body temperatures 3 times daily (at 9:00, 12:00 and 20:00 o'clock) and any symptoms such as cough, rhinorrhea, sore throat, fatigue, appetite loss, myalgia, vomiting, or diarrhea, occurring up to 5 days after the therapy started. Parents were requested to return the card by visiting or mailing to the clinic after completion of the course. Time until treatment was defined as days from fever onset until the clinic visit.

Nasopharyngeal swabs or aspirates were collected from the patients, placed in viral transport medium, and then transferred to the Department of Public Health, Niigata University Graduate School of Medical and Dental Sciences. The samples were stored at $4^{\circ} \mathrm{C}$ for a few days until viral culture, and aliquots were kept at $-80^{\circ} \mathrm{C}$. For virus isolation, supernatants of specimens were inoculated into Madin-Darby canine kidney cells. Types and subtypes were determined by hemagglutination inhibition tests with type-specific antisera (Masuda et al. 2000). Detection of the influenza genome was performed by reverse transcription-polymerase chain reaction (RT-PCR) (Saito et al. 2002). Briefly, viral RNA was extracted from nasopharyngeal aspirate specimens and reverse transcription reactions were performed for complementary DNA synthesis as described previously 
(Masuda et al. 2000). First and nested PCR was performed to detect generic influenza A, using M2 gene primers (Masuda et al. 2000). Influenza B was detected in separate PCR runs using influenza B hemagglutinin gene primers (Shimizu et al. 1997). In this study, we defined "influenza infections" as PCR or virus isolation positive regardless of rapid test results (Fig. 1). This study was approved by the Medical Faculty Ethics Committee of the Niigata University, Graduate School of Medical and Dental Sciences.

\section{Effectiveness of oseltamivir}

Influenza-related fever was defined as body temperature of more than $37.5^{\circ} \mathrm{C}(99.5 \mathrm{~F})$ using the highest body temperature among three different time measurements in a day. The effectiveness was evaluated by the fever duration more than $37.5^{\circ} \mathrm{C}$ after the first visit to clinic.

\section{Statistical analysis}

Statistical comparisons for baseline characteristics among the 4 groups by type of influenza and treatment were made by chi-square test to evaluate the proportions in multiple groups, and one-way analysis of variance to compare the mean values. Sheffe's test was used as univariate analysis to compare average values for the duration of fever among the four clinical groups. General linear model was employed as multi-variate analysis to assess independent variable which influenced the duration of fever and to estimate the adjusted average days for duration of fever by type and treatment. All statistical analyses were performed with SPSS 11.0J (SPSS Japan Inc., Tokyo). $P<0.05$ was considered statistically significant.

\section{Results}

\section{Patient characteristics}

A total of 1,848 individuals with influenzalike illness were screened during the four successive seasons for the study (Fig. 1). Among these, $1,130(61.1 \%)$ patients were positive for influenza with virus isolation or PCR, but nearly half of patients were excluded due to the reasons listed in the Fig. 1. As a result, a total of 602 patients (5 of

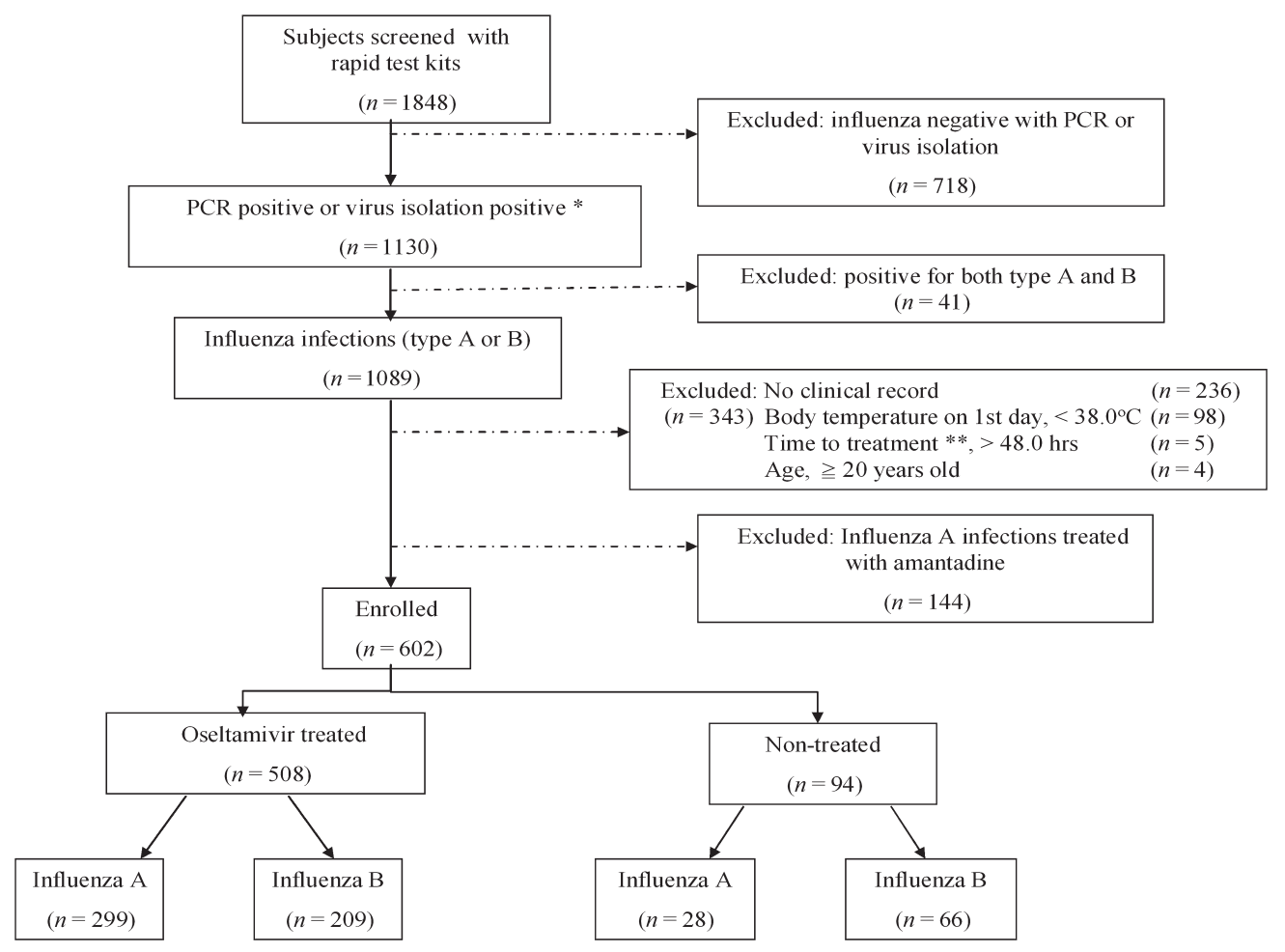

Fig. 1. Flow of participants through the study.

* Subjects were included regardless of rapid test results.

** Time until treatment, the time from the onset of fever to the first dose of treatment. 
influenza $\mathrm{A} / \mathrm{H} 1 \mathrm{~N} 1,257$ of $\mathrm{A} / \mathrm{H} 3 \mathrm{~N} 2$, and 257 of $\mathrm{B}$ were identified by virus isolation, and 65 of influenza A and 18 of influenza B by PCR) were enrolled in the study. They were divided into four groups by type of influenza and oseltamivir treatment status: 299 influenza A patients received oseltamivir treatment (treated influenza A), and 28 without treatment (non-treated influenza A), and 209 influenza B patients with treatment (treated influenza B) and 66 without treatment (non-treated influenza B), respectively (Table 1). The mean age and body weights, vaccination status, and the time until treatment did not differ significantly among the four groups. Body temperature at the time of clinic visit was higher in treated influenza A patients than treated influenza B, and younger patients ( $<6$ years old) had higher temperature than older ones ( $\geqq 6$ years old) in all groups.
Effectiveness of oseltamivir treatment for influenza $A$ and $B$

The duration of fever was shorter in the treatment group as compared to the non treatment in influenza A $(1.8 \pm 0.9$ days vs $2.6 \pm 1.3$ days; $p<0.01$ ), but influenza B did not have statistical significance $(2.4 \pm 1.3$ days vs $2.8 \pm 1.2$ days; $p=$ 0.09) (Table 2). The fever duration was longer for influenza B treatment group ( $2.4 \pm 1.3$ days) than influenza A treatment group (1.8 \pm 0.9 days; $p<$ $0.01)$. In all four groups, duration of fever was significantly longer in younger $(<6$ years old) than older children ( $\geqq 6$ years old) (Table 2 ). For younger group, the duration of fever was statistically shorter in treatment groups than non-treatment for both influenza A $(3.1 \pm 1.3$ days vs $1.9 \pm$ 1.0 days, $p<0.01$, balance between the two $=1.2$ days), and influenza B (3.2 \pm 1.1 days vs $2.7 \pm 1.3$ days, $p<0.05$, balance between the two $=0.5$ days), but not in older children for both influenza

Table 1. Demographic details for influenza A and B patients by oseltamivir treatment.

\begin{tabular}{|c|c|c|c|c|c|}
\hline & \multicolumn{2}{|c|}{ Influenza A } & \multicolumn{2}{|c|}{ Influenza B } & \multirow[b]{2}{*}{$p$ value $\mathrm{e}^{\mathrm{a}}$} \\
\hline & $\begin{array}{l}\text { Oseltamivir } \\
\text { non-treated } \\
\quad(n=28)\end{array}$ & $\begin{array}{l}\text { Oseltamivir } \\
\text { treated } \\
(n=299)\end{array}$ & $\begin{array}{c}\text { Oseltamivir } \\
\text { non-treated } \\
\quad(n=66)\end{array}$ & $\begin{array}{l}\text { Osertamivir } \\
\text { treated } \\
(n=209)\end{array}$ & \\
\hline Season & & & & & 0.000 \\
\hline 2001-2002 & $7(25.0)$ & $6(2.0)$ & $29(43.9)$ & $38(18.2)$ & \\
\hline $2002-2003$ & $6(21.4)$ & $28(9.4)$ & $0(0.0)$ & $6(2.9)$ & \\
\hline 2003-2004 & $2(7.1)$ & $109(36.5)$ & $0(0.0)$ & $4(1.9)$ & \\
\hline 2004-2005 & $13(46.4)$ & $156(52.2)$ & $37(56.1)$ & $161(77.0)$ & \\
\hline Gender & & & & & 0.036 \\
\hline Male & $9(32.1)$ & $175(58.5)$ & $39(59.1)$ & $109(52.2)$ & \\
\hline Female & $19(67.9)$ & $124(41.5)$ & 27 (40.9) & $100(47.8)$ & \\
\hline Age (years) & $4.9 \pm 4.0$ & $5.8 \pm 3.6$ & $5.7 \pm 2.3$ & $6.4 \pm 2.7$ & 0.044 \\
\hline Body temperature at the clinic visit $\left({ }^{\circ} \mathrm{C}\right)$ & $39.1 \pm 0.6$ & $39.2 \pm 0.6$ & $38.9 \pm 0.6$ & $39.0 \pm 0.6$ & 0.000 \\
\hline Body weight (kg) & $17.8 \pm 10.0$ & $22.5 \pm 11.6$ & $20.2 \pm 6.1$ & $22.4 \pm 9.4$ & 0.054 \\
\hline Time until treatment ${ }^{\mathrm{b}}$ (days) & $1.0 \pm 1.0$ & $0.8 \pm 0.6$ & $0.8 \pm 0.7$ & $0.9 \pm 0.7$ & 0.208 \\
\hline Vaccination & $7(25.0)$ & $112(37.5)$ & $17(25.8)$ & 83 (39.7) & 0.115 \\
\hline Use of antifebrile drug & $0(0.0)$ & $9(3.0)$ & $0(0.0)$ & $4(1.9)$ & 0.392 \\
\hline
\end{tabular}

Numbers are mean \pm S.D. or $n(\%)$.

${ }^{a}$ Chi-square test were employed for multiple rows and column contingency table, and one-way analysis of variance was used to compare means in multiple groups.

${ }^{\mathrm{b}}$ Time until treatment, the time from the onset of fever to the clinic visit. 
Oseltamivir Therapy for Children with Influenza

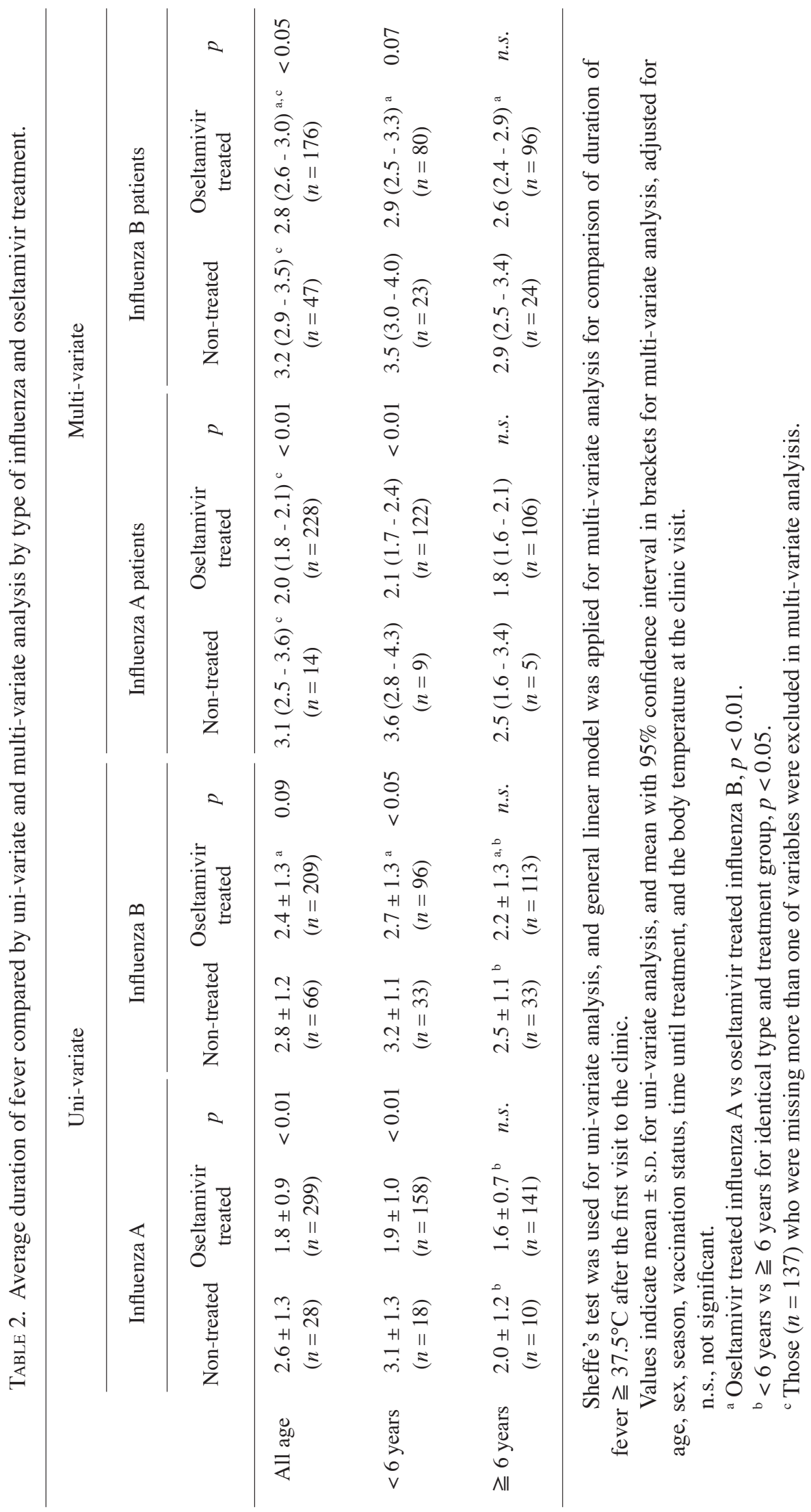


TABLE 3. Effects of influenza type, oseltamivir treatment, time until treatment, and maximum body temperature on the duration of fever analyzed with multi-variate analysis.

\begin{tabular}{lcc}
\hline \multicolumn{1}{c}{ Factor } & $\beta$ (day) & $p$ value \\
\hline Influenza B virus infection & 0.142 & 0.659 \\
Oseltamivir treatment & -1.321 & 0.000 \\
Age less than 6 years old & 0.711 & 0.011 \\
One degree higher body temperature at the clinic visit $\left({ }^{\circ} \mathrm{C}\right)$ & 0.550 & 0.000 \\
\hline
\end{tabular}

General linear model was carried out with 465 patients, adjusted for gender, body weight, season, vaccination status and the time until treatment. Those $(n=137)$ who were missing more than one of variables were excluded from the analysis.

A $(2.0 \pm 1.2$ days vs $1.6 \pm 0.7$ days, n.s., balance between the two $=0.4$ days $)$, and influenza B ( 2.5 \pm 1.1 days vs $2.2 \pm 1.3$ days, $n . s$., balance between the two $=0.3$ days). However, the fever duration was consistently shorter in treated influenza A than treated B for the two age categories.

We examined independent variable factors influencing the duration of fever using general linear model as multi-variate analysis (Table 3). Of variables analyzed, treatment of oseltamivir was a factor that attributed to the reduction of the fever duration by 1.32 days, whereas influenza $B$ virus infection did not affect the illness duration significantly. Patients who were less than 6 years old exhibited the prolonged duration of fever by 0.71 days, and as well as one degree higher body temperature at the clinic visit by 0.55 days.

Average duration of fever was estimated in the four groups with adjustment for age, gender, body weight, influenza season, vaccination status, time until treatment, and body temperature at the clinic visit. The treatment groups had significantly shorter duration of fever than non-treatment groups for both influenza A (2.0 days vs 3.1 days, $p<0.01)$ and influenza B (2.8 days vs 3.2 days, $p<0.05$ ) (Table 2). The duration was longer in treated influenza B than treated influenza A $(p<$ $0.01)$, as in the uni-variate analysis. After stratification by age groups ( $<6$ years old, or $\geqq 6$ years old), average duration was consistently longer for all four groups in younger children than older ones (Table 2$)$. In younger children $(<6$ years old), the fever duration was significantly shorter in treated groups than non- treated for influenza A
(3.6 days vs 2.1 days, $p<0.01$, balance between the two $=1.5$ days $)$, but not for influenza B (3.5 days vs 2.9 days, $p=0.74$, balance between the two $=0.6$ days $)$. In older children ( $\geqq 6$ years old), statistical significance was not demonstrated for both influenza A (2.5 days vs 1.8 days, n.s., balance between the two $=0.7$ days $)$ and $\mathrm{B}(2.9$ days vs 2.6 days, n.s., balance between the two $=$ 0.3 days). For the two age groups, treated influenza B had consistently longer fever duration than influenza A counterparts.

\section{Discussion}

The clinical results in this paper provided evidence that oseltamivir was effective in reducing the duration of fever for both influenza $A$ and $B$ infections, but was less effective for influenza $B$ infections rather than influenza A. Even after adjustment with various underlying factors, or categorization by age groups, the duration of fever in the treatment groups was consistently longer for influenza B than influenza A.

Oseltamivir has been thought to be equally effective against influenza $\mathrm{A}$ and $\mathrm{B}$ infections (Hayden et al. 1999; Whitley et al. 2001), but growing clinical evidence suggests oseltamivir is less effective against influenza B than influenza A. Our results were basically similar to the previous findings from Japan (Kawai et al. 2006; Sugaya et al. 2007). However, we emphasize that we carried out the study in multiple years, and enrolled sufficient number of non-treated groups for both influenza A and B in order to evaluate the effectiveness of the drug, in comparison with the pre- 
vious studies implemented in a single year, and included relatively limited number of non-treated patients (Kawai et al. 2006; Sugaya et al. 2007). In addition, multi-variate analysis was employed to estimate the most influencing factors for the duration of fever, on the top of uni-variate analysis, which might be affected by confounding factors.

In our study, adjusted average duration of fever showed that treated influenza B had longer clinical course than treated influenza A. In vitro data suggested that the $\mathrm{IC}_{50}$ of influenza $\mathrm{B}$ virus to oseltamivir was higher than influenza $\mathrm{A} / \mathrm{H} 3 \mathrm{~N} 2$ and A/H1N1 (Gubareva et al. 2001; Boivin and Goyette 2002; Hurt et al. 2004; Sugaya et al. 2007). Also, longer virus shedding was observed with influenza B than influenza A after oseltamivir treatment (Kawai et al. 2007). These data suggested that influenza B was less susceptible to oseltamivir than influenza $\mathrm{A}$ in vitro and in vivo. However, increasing the dosage for influenza B may not be advisable, since it prompts the issues of increased adverse effects. Choosing zanamivir for influenza B treatment is one of options (Kawai et al. 2008), but age limitations to this inhaling drug ( $\geqq 5$ years old) makes it difficult to generalize in pediatric practices.

Fever duration was consistently longer in younger children than older ones regardless of treatment. It is generally accepted that younger children with few previous influenza infections possessed prolonged course of illness and higher virus titer, due to insufficient inhibition of viral replication and higher cytokine levels. (Kiso et al. 2004; Kawai et al. 2008). In our analysis, oseltamivir seemed to be more effective in younger children than older children. It is true that insufficient number in some groups for older children (especially non-treated groups) made the results difficult to interpret, but statistical significance were more obvious in younger children than older ones. Also, the balance of fever duration between treated and non-treated was wider for younger children than older children for both influenza A and B. Despite the fact that the younger children had prolonged fever, the effect of treatment could be expected more in these groups. We need fur- ther investigations by enrolling larger number of children for confirmation.

In our multi-variate analysis, time from the onset to the clinic did not affect the fever duration as an independent variable. This is contrary to Kawai et al., reporting an increased benefit with early report to the clinic for the duration of fever (Kawai et al. 2008). These contrasting results were derived from the different criteria for fever duration between the two studies.

Higher body temperature at the first clinic visit was also a prolonging factor for fever duration as in the previous study (Kawai et al. 2008), suggesting the influences of higher viral replication and increased cytokines levels (Kiso et al. 2004; Kawai et al. 2008).

In conclusion, our study demonstrated the clinical effectiveness of oseltamivir for both influenza A and B patients, compared to non-treated patients. However, illness was prolonged for influenza B infections than influenza A under the treatment of oseltamivir.

\section{Acknowledgments}

This study was supported by Japan Grants-inAid for Scientific Research, from Monbu Kagakusho (Ministry of Education, Culture, Sports, Science and Technology, Japan), and Acute Respiratory Infections Panels, United States-Japan Cooperative Medical Science Program (U.S. Department of Health and Human Services, U.S. Department of State, United States, Ministry of Foreign Affairs, Ministry of Health, Labor, and Welfare, and Ministry of Education, Culture, Sports, Science and Technology, Japan). This study was presented at $80^{\text {th }}$ Japanese Association for Infectious Disease, 20-21 April, 2006, Tokyo, Japan. We thank Ms. Akemi Watanabe for technological assistance for virus isolation, and Ms. Yoshiko Kato for intensive secretarial work. We thank Mr. Clyde Pancit Dapat and Dr. Shamsul Azhar Shah for reviewing the manuscript.

\section{References}

Aoki, F.Y., Macleod, M.D., Paggiaro, P., Carewicz, O., El Sawy, A., Wat, C., Griffiths, M., Waalberg, E. \& Ward, P. (2003) Early administration of oral oseltamivir increases the benefits of influenza treatment. J. Antimicrob. Chemother., 51, 123-129.

Boivin, G. \& Goyette, N. (2002) Susceptibility of recent Canadian influenza A and B virus isolates to different neuraminidase inhibitors. Antiviral Res., 54, 143-147.

Centers for Disease Control and Prevention (2006) Prevention 
and Control of Influenza. Recommendations of the Advisory Committee on Immunization Practices (ACIP). MMWR, 55, 24-30 (Also available at http://www.cdc.gov/mmwr/ $\mathrm{PDF} / \mathrm{rr} / \mathrm{rr} 5510 . \mathrm{pdf})$.

Gubareva, L.V., Webster, R.G. \& Hayden, F.G. (2001) Comparison of the activities of zanamivir, oseltamivir, and RWJ-270201 against clinical isolates of influenza virus and neuraminidase inhibitor-resistant variants. Antimicrob. Agents Chemother., 45, 3403-3408.

Hayden, F.G., Treanor, J.J., Fritz, R.S., Lobo, M., Betts, R.F., Miller, M., Kinnersley, N., Mills, R.G., Ward, P. \& Straus, S.E. (1999) Use of the oral neuraminidase inhibitor oseltamivir in experimental human influenza: randomized controlled trials for prevention and treatment. JAMA, 282, 1240-1246.

Hurt, A.C., McKimm-Breschkin, J.L., McDonald, M., Barr, I.G., Komadina, N. \& Hampson, A.W. (2004) Identification of a human influenza type B strain with reduced sensitivity to neuraminidase inhibitor drugs. Virus Res., 103, 205-211.

Jefferson, T., Demicheli, V., Rivetti, D., Jones, M., Di Pietrantonj, C. \& Rivetti, A. (2006) Antivirals for influenza in healthy adults: systematic review. Lancet, 367, 303-313.

Kawai, N., Ikematsu, H., Iwaki, N., Satoh, I., Kawashima, T., Maeda, T., Miyachi, K., Hirotsu, N., Shigematsu, T. \& Kashiwagi, S. (2005) Factors influencing the effectiveness of oseltamivir and amantadine for the treatment of influenza: a multicenter study from Japan of the 2002-2003 influenza season. Clin. Infect. Dis., 40, 1309-1316.

Kawai, N., Ikematsu, H., Iwaki, N., Maeda, T., Satoh, I., Hirotsu, N. \& Kashiwagi, S. (2006) A comparison of the effectiveness of oseltamivir for the treatment of influenza A and influenza B: a Japanese multicenter study of the 2003-2004 and 2004-2005 influenza seasons. Clin. Infect. Dis., 43, 439-444.

Kawai, N., Ikematsu, H., Iwaki, N., Kawashima, T., Maeda, T., Mitsuoka, S., Kondou, K., Satoh, I., Miyachi, K., Yamaga, S., Shigematsu, T., Hirotsu, N. \& Kashiwagi, S. (2007) Longer virus shedding in influenza B than in influenza A among outpatients treated with oseltamivir. J. Infect., 55, 267-272.

Kawai, N., Ikematsu, H., Iwaki, N., Maeda, T., Kanazawa, H., Kawashima, T., Tanaka, O., Yamauchi, S., Kawamura, K., Nagai, T., Horii, S., Hirotsu, N. \& Kashiwagi, S. (2008) A comparison of the effectiveness of zanamivir and oseltamivir for the treatment of influenza A and B. J. Infect., 56, 51-57.

Kiso, M., Mitamura, K., Sakai-Tagawa, Y., Shiraishi, K., Kawakami, C., Kimura, K., Hayden, F.G., Sugaya, N. \& Kawaoka, Y. (2004) Resistant influenza A viruses in children treated with oseltamivir: descriptive study. Lancet, 364, 759-765.

Masuda, H., Suzuki, H., Oshitani, H., Saito, R., Kawasaki, S., Nishikawa, M. \& Satoh, H. (2000) Incidence of amanta- dine-resistant influenza A viruses in sentinel surveillance sites and nursing homes in Niigata, Japan. Microbiol. Immunol., 44, 833-839.

Monto, A.S. (2003) The role of antivirals in the control of influenza. Vaccine, 21, 1796-1800.

Moscona, A. (2005) Neuraminidase inhibitors for influenza. $N$. Engl. J. Med., 353, 1363-1373.

Mungall, B.A., Xu, X. \& Klimov, A. (2004) Surveillance of influenza isolates for susceptibility to neuraminidase inhibitors during the 2000-2002 influenza seasons. Virus Res., 103, 195-197.

Nicholson, K.G., Aoki, F.Y., Osterhaus, A.D., Trottier, S., Carewicz, O., Mercier, C.H., Rode, A., Kinnersley, N. \& Ward, P. (2000) Efficacy and safety of oseltamivir in treatment of acute influenza: a randomised controlled trial. Neuraminidase Inhibitor Flu Treatment Investigator Group. Lancet, 355, 1845-1850.

Nicholson, K.G., Wood, J.M. \& Zambon, M. (2003) Influenza. Lancet, 362, 1733-1745.

Oxford, J.S., Bossuyt, S., Balasingam, S., Mann, A., Novelli, P. $\&$ Lambkin, R. (2003) Treatment of epidemic and pandemic influenza with neuraminidase and M2 proton channel inhibitors. Clin. Microbiol. Infect., 9, 1-14.

Oxford, J.S. (2005) Preparing for the first influenza pandemic of the 21st century. Lancet Infect. Dis., 5, 129-131.

Saito, R., Oshitani, H., Masuda, H. \& Suzuki, H. (2002) Detection of amantadine-resistant influenza A virus strains in nursing homes by PCR-restriction fragment length polymorphism analysis with nasopharyngeal swabs. J. Clin. Microbiol., 40, 84-88.

Shimizu, H., Watanabe, S. \& Imai, M. (1997) Rapid detection of influenza virus A (AH1, AH3) and B by nested-polymerase chain reaction. Kansenshogaku Zasshi, 71, 522-526. (in Japanese)

Simonsen, L., Fukuda, K., Schonberger, L.B. \& Cox, N.J. (2000) The impact of influenza epidemics on hospitalizations. $J$. Infect. Dis., 181, 831-837.

Sugaya, N., Mitamura, K., Yamazaki, M., Tamura, D., Ichikawa, M., Kimura, K., Kawakami, C., Kiso, M., Ito, M., Hatakeyama, S. \& Kawaoka, Y. (2007) Lower clinical effectiveness of oseltamivir against influenza B contrasted with influenza A infection in children. Clin. Infect. Dis., 44, 197-202.

Treanor, J.J., Hayden, F.G., Vrooman, P.S., Barbarash, R., Bettis, R., Riff, D., Singh, S., Kinnersley, N., Ward, P. \& Mills, R.G. (2000) Efficacy and safety of the oral neuraminidase inhibitor oseltamivir in treating acute influenza: a randomized controlled trial. US Oral Neuraminidase Study Group. JAMA, 283, 1016-1024.

Whitley, R.J., Hayden, F.G., Reisinger, K.S., Young, N., Dutkowski, R., Ipe, D., Mills, R.G. \& Ward, P. (2001) Oral oseltamivir treatment of influenza in children. Pediatr. Infect. Dis. J., 20, 127-133. 